\title{
Nanometric Diamond Delta Doping with Boron
}

\author{
James E. Butler ${ }^{1,2 *}$, Anatoly Vikharev ${ }^{1}$, Alexei Gorbachev ${ }^{1}$, Mikhail Lobaev ${ }^{1}$, Anatoly Muchnikov ${ }^{1}$, Dmitry \\ Radischev ${ }^{1}$, Vladimir Isaevv ${ }^{1}$, Valerii Chernov ${ }^{1}$, Sergey Bogdanov ${ }^{1}$, Mikail Drozdov ${ }^{3}$, Evgeniy Demidov ${ }^{3}$, \\ Ekaterina Surovegina ${ }^{3}$, Vladimir Shashkin ${ }^{3}$, Albert Davidov ${ }^{4}$, Haiyan $\operatorname{Tan}^{4}$, Louisa Meshi ${ }^{4,5}$, Alexander C. \\ Pakpour-Tabrizi ${ }^{6}$, Marie-Laure Hicks ${ }^{6}$, Richard B. Jackman ${ }^{6}$ \\ ${ }^{1}$ Institute of Applied Physics, Russian Academy of Sciences, Nizhny Novgorod, Russia \\ ${ }^{2}$ St. Petersburg Electrotechnical University (LETI), St. Petersburg, Russia \\ ${ }^{3}$ Institute for Physics of Microstructures of the Russian Academy of Sciences, Nizhny Novgorod, Russia \\ ${ }^{4}$ National Institute of Standards and Technology, Materials Science and Engineering Division, Gaithersburg MD, USA \\ ${ }^{5}$ Ben Gurion University, Department of Materials Engineering, Beersheba, Israel \\ ${ }^{6}$ London Centre for Nanotechnology and the Department of Electronic and Electrical Engineering, University College London, \\ UK
}

Keywords: Diamond, Boron, Nanometer, Delta Doping, Mobility

\begin{abstract}
:
Diamond is desired for active semiconducting device because of it high carrier mobility, high voltage breakdown resistance, and high thermal diffusivity. Exploiting diamond as a semiconductor is hampered by the lack of shallow dopants to create sufficient electronic carriers at room temperature. In this work, nanometer thick, heavily boron doped epitaxial diamond 'delta doped' layers have been grown on ultra smooth diamond surfaces which demonstrate $\mathrm{p}$ type conduction with enhanced Hall mobilities of up to $120 \mathrm{~cm}^{2} / \mathrm{Vs}$ and sheet carrier concentrations to $6 \times 10^{13} \mathrm{~cm}^{-2}$, thus enabling a new class of active diamond electronic devices.
\end{abstract}

Diamond is desired as a material for high voltage, high frequency, high power active and passive electronic devices because of its superlative materials properties ${ }^{[1]}$, including high electronic carrier mobilities ${ }^{[2]}$, high breakdown field strength ${ }^{[3]}$, high thermal diffusivity ${ }^{[4]}$, matrix for quantum devices, and diverse other optical, chemical, materials properties ${ }^{[5]}$. However, a major barrier to exploiting diamond for active electronic applications has been the lack of dopants with a sufficiently low thermal activation energy barrier to create an adequate concentration of electronic carriers at room temperature. While there are many known defect and impurities states in the wide bandgap $(5.45 \mathrm{eV})$ of diamond, several of which can act as donors or acceptors of electronic charge, only boron ${ }^{[1,6]}$ (creating an acceptor state) and phosphorous (creating a donor state) ${ }^{[7]}$ have been demonstrated as reliable dopants. Boron, the most commonly used diamond dopant, has the smallest activation energy of $0.37 \mathrm{eV}$ at low doping concentrations $\left(<10^{17} \mathrm{~cm}^{-3}\right)$. This value still means that only a fraction of the boron present is activated at room temperature leading to relatively low concentrations of free carriers. Increasing the boron concentration reduces this activation energy, and at ca. $5 \times 10^{20} \mathrm{~cm}^{-3}$ the metal-to-insulator transition point occurs ${ }^{[8]}$ and a fullyactivated impurity band is formed via the quantum tunneling of holes between neighboring boron acceptor states ${ }^{[9]}$. Unfortunately, as the activation energy of holes decreases so does carrier mobility, not only because of the increased impurity scattering but also due to the onset of a low-mobility, hopping-like conduction. The resulting material is one that has sub-unity carrier mobility and typical sheet carrier densities in excess of that which are readily controlled by a typical field effect transistor $(\mathrm{FET})^{[10]}$. In this work we demonstrate two-dimensional conduction 
with both high mobility and sheet carrier concentration by introducing nanometer thick boron 'delta doped layers' into intrinsic or $\mathrm{p}^{-}$material ( $\mathrm{p}^{-}$here defined as a boron concentration of less than $10^{17} \mathrm{~cm}^{-3}$ ) epitaxially grown diamond films with high mobility.

A well-known solution to creating BOTH high mobility AND high carrier concentrations for electronic materials in two dimensions is 'nanometric delta doped' layers ${ }^{[1]]}$ : nanometer thick, heavily doped layers (boron in

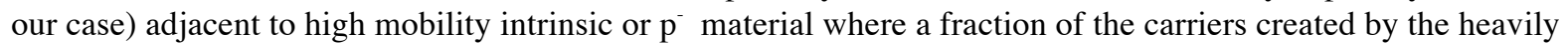
ionized dopant layer reside in the adjacent high mobility layer. Similar two-dimensional carrier conductivity has been observed on hydrogen terminated diamond surfaces with negative electron affinity. These surfaces create a subsurface two-dimensional hole gas ${ }^{[12]}$ by electron transfer to adsorbates, but since they display stability issues, they will not be discussed further in this work.

The success of 'delta doping' requires the epitaxial growth of very thin (1 to $2 \mathrm{~nm}$ ) heavily doped 'delta layer' (preferably above the insulator to metal transition, ca. $5 \times 10^{20} \mathrm{~cm}^{-3}$ for boron in diamond) ${ }^{[8]}$ and abrupt interfaces between the 'delta layer' and the high mobility intrinsic or $\mathrm{p}^{-}$layer ${ }^{[13]}$ In addition, the interfaces must be atomically smooth to minimize carrier scattering.

Recent reports from three experimental studies provide an in depth discussion of their observations and implications. Balmer et al. ${ }^{[14]}$ interpret measurements on uncapped, surface boron doped 'delta layers' with a two carrier type model, but present no data showing the expected enhanced mobilities and sheet carrier concentrations. They also point out the mobilities measured by the Hall effect will overestimate the channel mobility in a working device. Scharpf et al. ${ }^{[15]}$ demonstrate a boron doped solution gated field effect transistor based on an uncapped surface 'delta layer', with adequate sheet carrier density, but a channel mobility of $0.1 \mathrm{~cm}^{2} / \mathrm{Vsec}$ which they attribute to poor lateral homogeneity and interrupted morphology of the 'delta layers'. Chicot et al. ${ }^{[16]}$ present results on buried boron doped 'delta layers' of varying thicknesses ranging from $<2$ to $35 \mathrm{~nm}$ which were capped with 30 to $65 \mathrm{~nm}$ of undoped diamond. They also summarize the published literature reports of mobilities and sheet carrier concentrations along with their results (ref. ${ }^{[16]}$, Table IV), which are reproduced in Table 1 below. All of their measured carrier mobilities were in the range of 1 to $4.4 \mathrm{~cm}^{2} / \mathrm{Vsec}$ which is typical of bulk diamond doped above the metal insulator transition of $5 \times 10^{20} \mathrm{~cm}^{-3}$. The desired values in 'delta doped' layers are greater than $100 \mathrm{~cm}^{2} / \mathrm{Vsec}$ for mobility, and $>10^{13} \mathrm{~cm}^{-2}$ for sheet carrier concentrations.

In this report, significant progress in growth and characteristics of diamond nanometric thick 'delta doping' with boron is shown, demonstrating both enhanced room temperature Hall effect mobilities and carrier concentrations over the previous experimental reports. The adopted approach employs: ultra-smooth diamond substrates $\left(\mathrm{R}_{\mathrm{a}} / \mathrm{S}_{\mathrm{a}}\right.$ less than $\left.0.3 \mathrm{~nm}\right)\left(\mathrm{R}_{\mathrm{a}}\right.$ is the arithmetic average of surface profile along a line, and $\mathrm{S}_{\mathrm{a}}$ is the average over an area), a custom built microwave plasma assisted chemical vapor deposition (CVD) reactor with laminar flow and fast gas switching ${ }^{[17]}$, very slow growth rates, smooth epitaxial films, and chemically gettering residual boron in the reactor when growing intrinsic/lightly doped material by adding $\mathrm{H}_{2} \mathrm{~S}$ to form gaseous boron sulfur compounds. ${ }^{[18]}$

\section{Experimental:}

The single crystal diamond substrates used in these experiment were type IIa (no nitrogen detectable by IR absorption spectroscopy) obtained from New Diamond Technology (St. Petersburg Russia) and the Technological Institute for Superhard and Novel Carbon Materials (Troitsk, Moscow, Russia). Their size was either 3x3x0.5 mm or $3.5 \times 3.5 \times 0.5 \mathrm{~mm}$ with all polished faces nominally (100). The samples displayed little or no birefringence when viewed between crossed polarizers. These samples were repolished mechanically to a surface roughness, $R_{a}$, of 0.3 $\mathrm{nm}$ or less, typically $0.1 \mathrm{~nm}$ over areas of $80 \times 80$ microns for $S_{a}$, and lines of over $1 \mathrm{~mm}$ for $R_{a}$, and then etched in an inductively coupled reactive ion etcher (Oxford Instruments, Plasmalab 80) to remove polishing damage in the top ca. 4.5 microns. ${ }^{[19]}$ The CVD reactor was a custom designed ${ }^{[17]}$ specifically for the 'delta doping' growths with nearly laminar flow and rapid switching of the reactant gas mixtures to achieve abrupt interfaces. Typical growth conditions employed a flow of $900 \mathrm{sccm}$ hydrogen (Pd diffusion cell purified), $1.4 \mathrm{sccm}$ methane (ultra pure, 99.999\%), 6 to $17 \mathrm{sccm}$ of $0.1 \% \mathrm{~B}_{2} \mathrm{H}_{6}$ diluted in hydrogen, and 6 to $14 \mathrm{sccm}$ of $0.1 \% \mathrm{H}_{2} \mathrm{~S}$ diluted in hydrogen. Typical growth rates were determined by SIMS-SP, see Figure 1, to be between 30 and $90 \mathrm{~nm} /$ hour. The total gas pressure was 30 to 50 torr and the microwave power was $1.5 \mathrm{~kW}$. Transmission electron microscope (TEM) images were recorded aligned to the diamond [110] axis. TEM sample preparation was performed in Helios FEI FIB. In order to keep the surface layer undamaged, it was coated by Platinum. TEM investigation was carried out on $300 \mathrm{kV}$ FEI Titan. SIMS-SP was performed in an IONTOF TOF.SIMS-5 using Cs+ ions $(1 \mathrm{keV})$ for sputtering, and Bi+ ions $\left(25 \mathrm{keV}\right.$ ) for probing. It was found out that for smooth (root mean square deviation $\mathrm{R}_{\mathrm{a}}<1 \mathrm{~nm}$ ) surfaces the 
using of $1 \mathrm{keV}$ energy sputtering ions allows to obtain depth resolution of 1.2-2 nm for the most of investigated structures.

Room temperature Hall measurements were carried out in van der Pauw geometry after by an acid cleaning process (boiling in conc. sulfuric and nitric acids with added $\mathrm{KNO}_{3}$ ) known to leave oxygen moieties on the diamond surface to minimize any contribution from a hydrogen surface conductive channel. At IPM, Indium Ohmic contacts were deposited at 180 degrees Celsius, not only on the surface but also to the end pads. This provided satisfactory electrical contacts to the delta layer with stable characteristics without additional annealing and allows the Hall measurements with small errors. At UCL, Ohmic contacts were made with silver epoxy annealed at $70 \mathrm{C}$. Measurements were made in a Lakeshore 7500 series Hall effect probe or a homemade system with stabilized current source $10^{-6}-10^{-3}$ A. Measurements were performed in magnetic fields of up to $1 \mathrm{~T}$ with both polarities.

Capacitance voltage measurements were performed on sample Tr06 with two 'delta layers' and an underlying heavily doped layer. Mesa structures of diameters 25 to 400 microns were formed by lithographic masking and etching down to the heavily boron doped layer. Ohmic contacts were formed after etching on the heavily doped layer $\mathrm{p}++$ layer by deposition of $\mathrm{Ti} / \mathrm{Mo} / \mathrm{Au}$ metallization and annealing. Schottky contacts were formed on the top of the mesas with $\mathrm{Cr} / \mathrm{Al}$ metallization. Measurement of static current-voltage characteristics was performed using a Keithley 4200-SCS system. The capacitance-voltage characteristics were measured on the instrument Agilent E4980A Precision LCR Meter, over the frequency range $20 \mathrm{~Hz}$ to $2 \mathrm{MHz}$, with a $50 \mathrm{mV}$ amplitude of the test signal. For CV-profiling was performed at a frequency for which no observed frequency dispersion was observed in our structures $(<100 \mathrm{kHz})$.

Figure 1 (a) presents the boron concentration as a function of depth from the final surface measured by secondary ion mass spectrometry sputter profiling (SIMS-SP) for a 3 'delta layer' structure used as an example in determining growth rates and peak boron incorporation rates. Analysis of the boron profiles of delta-layers by SIMS was carried out in the mode of maximum dynamic range. This mode provides the best resolution in depth. However, the noise level in this mode is more than $4 \times 10^{17} \mathrm{~cm}^{-3}$. Well separated 'delta layers' of approximately $2 \mathrm{~nm}$ width are shown, capped and separated by 20 to $30 \mathrm{~nm}$ of $\mathrm{p}^{-}$doped diamond with boron levels below $2 \times 10^{17} \mathrm{~cm}^{-3}$. To determine the level of background boron doping between the layers more accurately, we used another mode with longer signal accumulation and a slow sputtering rate. Figure 1(b) shows the SIMS depth profile from a sample with a surface delta-layer, and similarly grown $\mathrm{p}^{-}$buffer layer over $200 \mathrm{~nm}$ thick. As can be seen from the figure, the background boron concentration in the $\mathrm{p}^{-}$grown layer is less than $3 \times 10^{16} \mathrm{~cm}^{-3}$, and the peak concentration in this delta layer exceeded $6 \times 10^{20} \mathrm{~B} \mathrm{~cm}^{-3}$.

Figure 1(c) presents the results of deconvoluting the SIMS-SP profile of the boron concentration of a single 'delta layer' of thickness ca. $2 \mathrm{~nm}$ as a function of the depth from the surface using a SIMS-SP instrument function ${ }^{\text {[20] }}$. The resolution of SIMS-SP is limited by many factors ${ }^{[21]}$ and may not be sufficient to show the abruptness of the interfaces. 
(a)

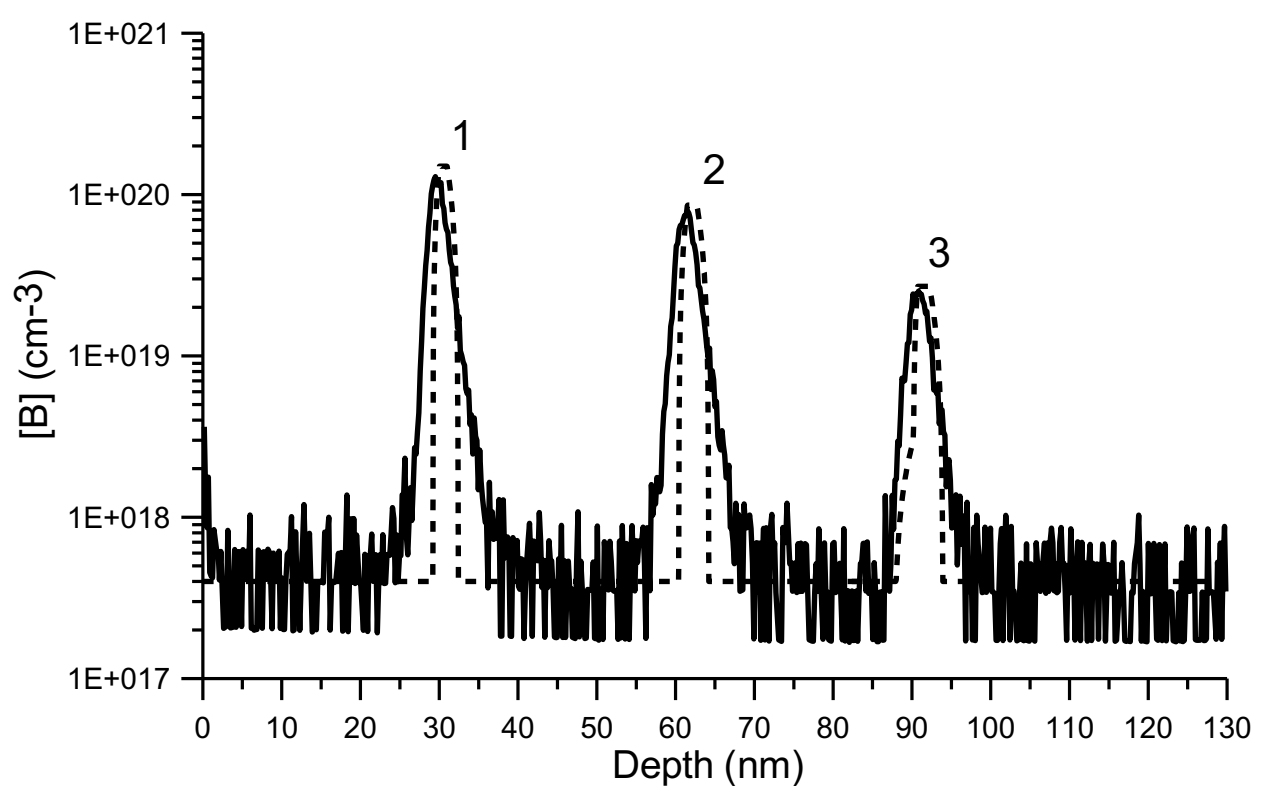

(b)

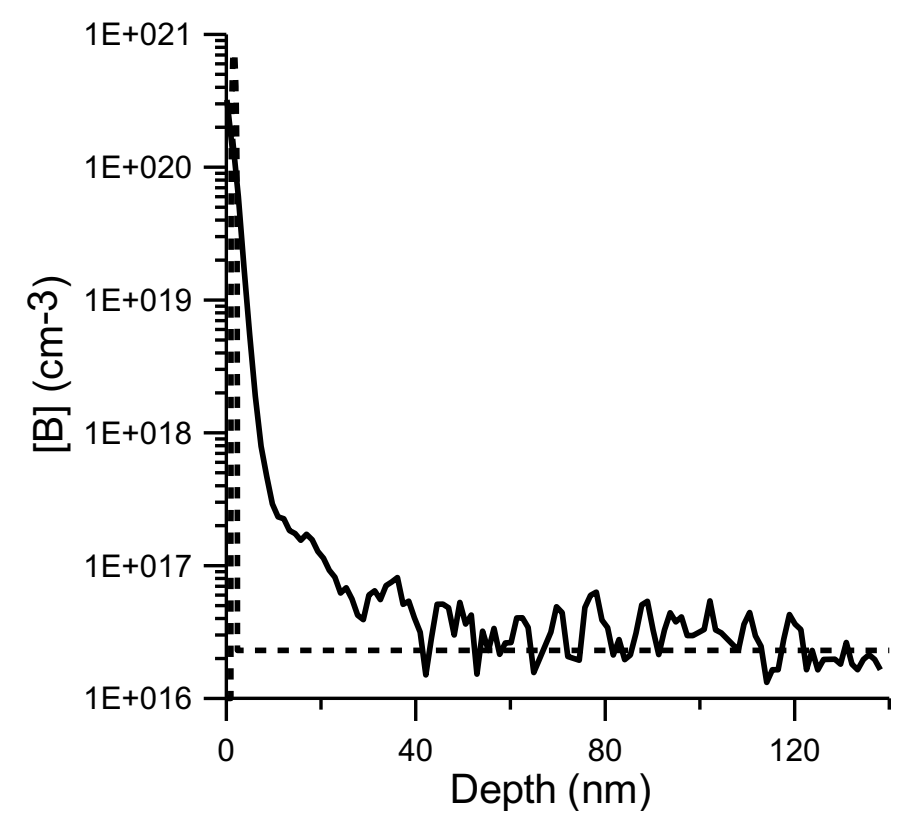

(c) 


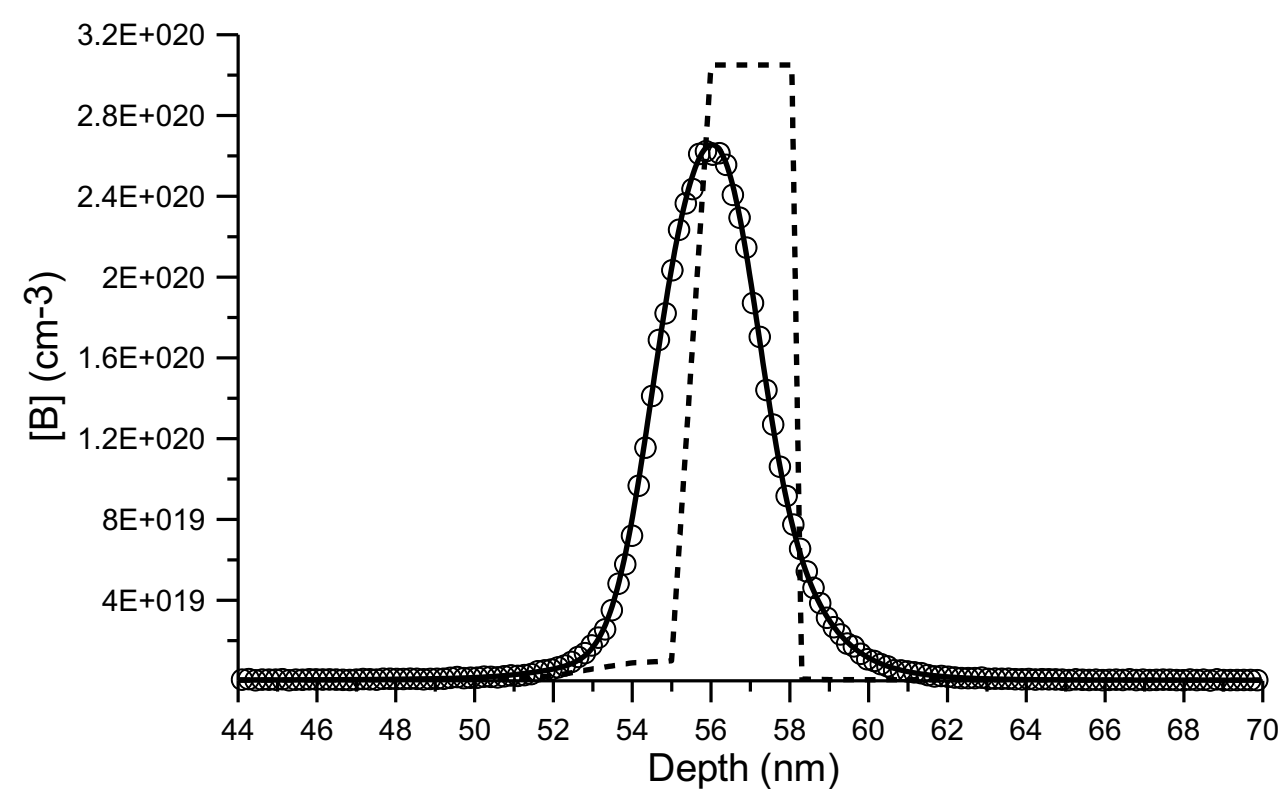

Figure 1. (a) Boron concentration profiles determined by SIMS-SP (solid line); Boron concentration profiles recovered using analytical depth resolution function (dash line). Methane flow is $1.4 \mathrm{sccm}$. B/C ratio $18570 \mathrm{ppm}(1)$, $12860 \mathrm{ppm}$ (2), $8570 \mathrm{ppm}$ (3). The profile is for sample S32. (b) Boron concentration profiles determined by SIMS$\mathrm{SP}$ (solid line) for an uncapped delta layer grown on $200 \mathrm{~nm} \mathrm{p}^{-}$epilayer, sample S38. Boron concentration profiles recovered using analytical depth resolution function (dash line), thickness is $1.2 \mathrm{~nm}$ and maximum concentration is 7 $\mathrm{x} 10^{20} \mathrm{~cm}^{-3}$. (c) SIMS-SP profile of a single 'delta layer' (open circles), Sample S27, and the boron concentration profiles recovered using analytical depth resolution function (dash line), and resulting fit profile (solid line). 
Examination of focused ion beam (FIB) thinned portions of one of our 'delta doped' samples by electron diffraction and transmission electron microscopy (TEM) confirms the [001] direction of our epitaxial growth, as shown in figure 2. It should be noted that due to the epitaxial relationship, similarity in atomic numbers of $\mathrm{C}$ (12) and B (13), maximum boron concentration of less than $0.1 \%$, and lack of significant structural defects, the interfaces between the buffer layer, substrate, 'delta layer', and cap layer are not visible in the figure. Shown in the figure are the positions expected for the epitaxial layers based on the expected growth rates of each layer. At lower magnifications (not shown) some additional contrast does appear in the 'delta doped' area, probably due to strain.

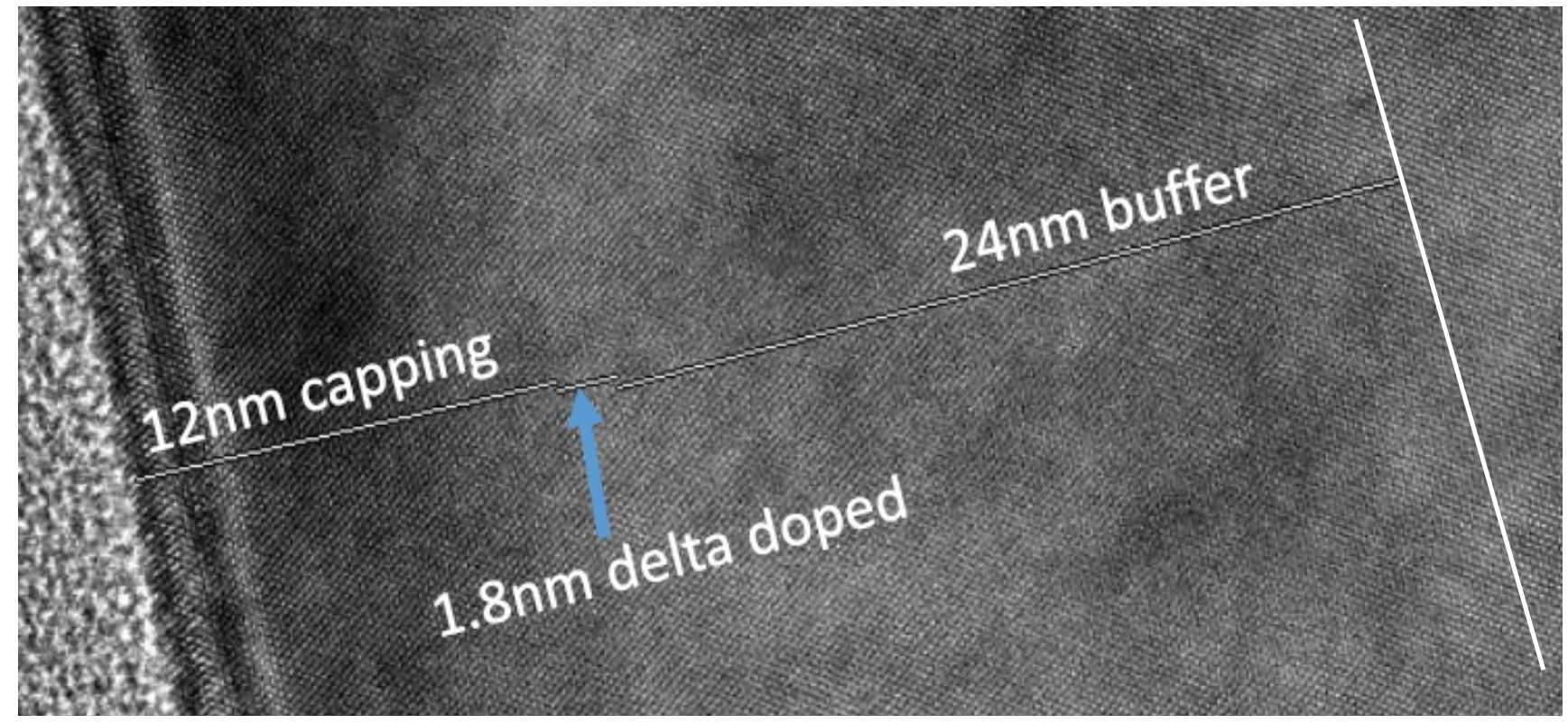

Figure 2: A transmission electron micrograph image of a focused ion beam thinned section of a sample with a single 'delta layer' sandwiched between intrinsic/lightly doped buffer and capping layers on a single crystal substrate. The electron beam was normal to the [110] crystallographic direction and the epitaxial growth direction was normal to the (001) crystallographic plane. The annotation of the layer positions is solely based on the expected growth rates (see text for explanation). 
The ultimate test of the success of 'delta doping' has to be in electrical measurements. We report here our initial room temperature measurements of carrier concentrations and mobilities by the Hall effect (van der Pauw configuration) on the whole samples containing single delta layers of thickness between 1.8 and $2.8 \mathrm{~nm}$ and capped with a buffer layer. Capacitance - voltage (CV) depth profiling of mesa isolated structure has also been measured. The results of measurements of the room temperature Hall effect mobilities and carrier concentrations using the van der Pauw geometry of our initial set of samples by 2 participating laboratories is reported in Table 1. Also reported in this table are the published measurements of several previous experimental efforts. The most notable thing is that the carrier mobilities of the samples grown in this work exceed those of all the previous reports on similar samples by over an order of magnitude (the non-italicized entries in table 1), and the related resistances are also over an order of magnitude lower. The italicized entries are for data which may be compromised by contributions dominated by the bulk, lightly doped regions of the sample and not as a result of conduction adjacent to the delta doped layer as discussed later.

Table 1: presents the sheet carrier concentrations, room temperature mobilities, and resistance of the samples grown for this work and measured at UCL and IPM. Additional measurements reported in the literature are also presented. Entries shown in italics are not directly relevant as the conduction is likely dominated by the bulk pbuffer and cap regions based on the thermal activation energy measurement as discussed in the referenced papers.

\begin{tabular}{|c|c|c|c|c|}
\hline source & $\begin{array}{l}\text { sample } \\
\text { label }\end{array}$ & $\begin{array}{l}\text { Ps } \\
\mathrm{cm}^{-2}\end{array}$ & $\begin{array}{l}\mathrm{T}=300 \mathrm{~K} \\
\mathrm{~cm}^{2} / \mathrm{Vs}\end{array}$ & $\begin{array}{l}\text { resistance } \\
\mathrm{ohm} / \mathrm{sq}\end{array}$ \\
\hline$U C L$ & S16 & $5.00 E+11$ & 750 & 16455 \\
\hline UCL & S19 & $1.00 \mathrm{E}+12$ & 90 & 33131 \\
\hline UCL & $\mathrm{S} 18$ & $4.00 \mathrm{E}+12$ & 10 & 182310 \\
\hline IPM & S09 & $6.50 \mathrm{E}+11$ & 240 & 40000 \\
\hline IPM & $\mathrm{S} 21$ & $6.50 \mathrm{E}+13$ & 16 & 6600 \\
\hline IPM & $\mathrm{S} 23$ & $1.00 \mathrm{E}+13$ & 99 & 6000 \\
\hline IPM & E35-14 & $1.30 \mathrm{E}+13$ & 120 & 4000 \\
\hline IPM & $\mathrm{S} 24$ & $6.40 \mathrm{E}+13$ & 92 & 1100 \\
\hline IPM & $\mathrm{S} 12$ & $5.10 \mathrm{E}+13$ & 17 & 7000 \\
\hline IPM & S12-1 & $6.00 \mathrm{E}+13$ & 18 & 5300 \\
\hline Ref. $^{[22]}$ & 1 & $1.40 E+13$ & 54.6 & \\
\hline Ref. $^{[22]}$ & 2 & $1.60 \mathrm{E}+14$ & 3.3 & 158000 \\
\hline Ref. $^{[22]}$ & 3 & $4.40 \mathrm{E}+15$ & 2.9 & \\
\hline Ref. $^{[22]}$ & 4 & $2.30 \mathrm{E}+15$ & 4.4 & \\
\hline Ref. & 5 & $3.20 \mathrm{E}+14$ & 3.3 & 15000 \\
\hline $\operatorname{Ref}^{[16]}$ & 6 & $3.20 \mathrm{E}+13$ & 1 & \\
\hline Ref. ${ }^{[16]}$ & 7 & $1.40 \mathrm{E}+14$ & 3.8 & 70000 \\
\hline Ref. ${ }^{[16]}$ & 8 & $1.80 \mathrm{E}+14$ & 3.1 & \\
\hline Ref. ${ }^{[16]}$ & 9 & $6.10 \mathrm{E}+14$ & 3.7 & \\
\hline Ref. ${ }^{[16]}$ & 10 & $2.20 \mathrm{E}+13$ & 0.6 & \\
\hline Ref. $^{[14]}$ & 348 & $3.70 E+12$ & 35.4 & \\
\hline $\operatorname{Ref.}^{[14]}$ & 366 & $1.00 E+16$ & 3.8 & \\
\hline $\operatorname{Ref}^{[14]}$ & 385 & $1.20 E+11$ & 679.8 & \\
\hline
\end{tabular}




$\begin{array}{llrrrr}\text { Ref. } .^{[14]} & & 459 & 4.30 E+08 & 438.1 & 126000 \\ \text { Ref. } .^{[23]} & A & & 1.40 E+15 & & \\ \text { Ref. } .^{[23]} & B & 8.00 E+12 & 9.8 & \\ {\text { Ref } .^{[23]}} \text { C } & 1.20 E+13 & 13.3 & \\ \text { Ref. }^{[15]} & \mathrm{S} & 2.40 \mathrm{E}+13 & 0.1 & \end{array}$

Figure 3 (a) presents a SIMS-SP of the boron concentration in sample Tr06 where 2 separate 'delta layers' were grown over an intrinsic/lightly doped buffer layer on a heavily boron doped layer. Figure 3(b) presents the results of capacitance versus voltage measurements on mesa structures showing the apparent profile of the hole concentration in one 'delta layer'. Note that the measured hole concentration outside the 'delta layer' is more than an order of magnitude above the residual boron concentration measured by SIMS-SP of less than $4 \times 10^{17} \mathrm{~cm}^{-3}$. 


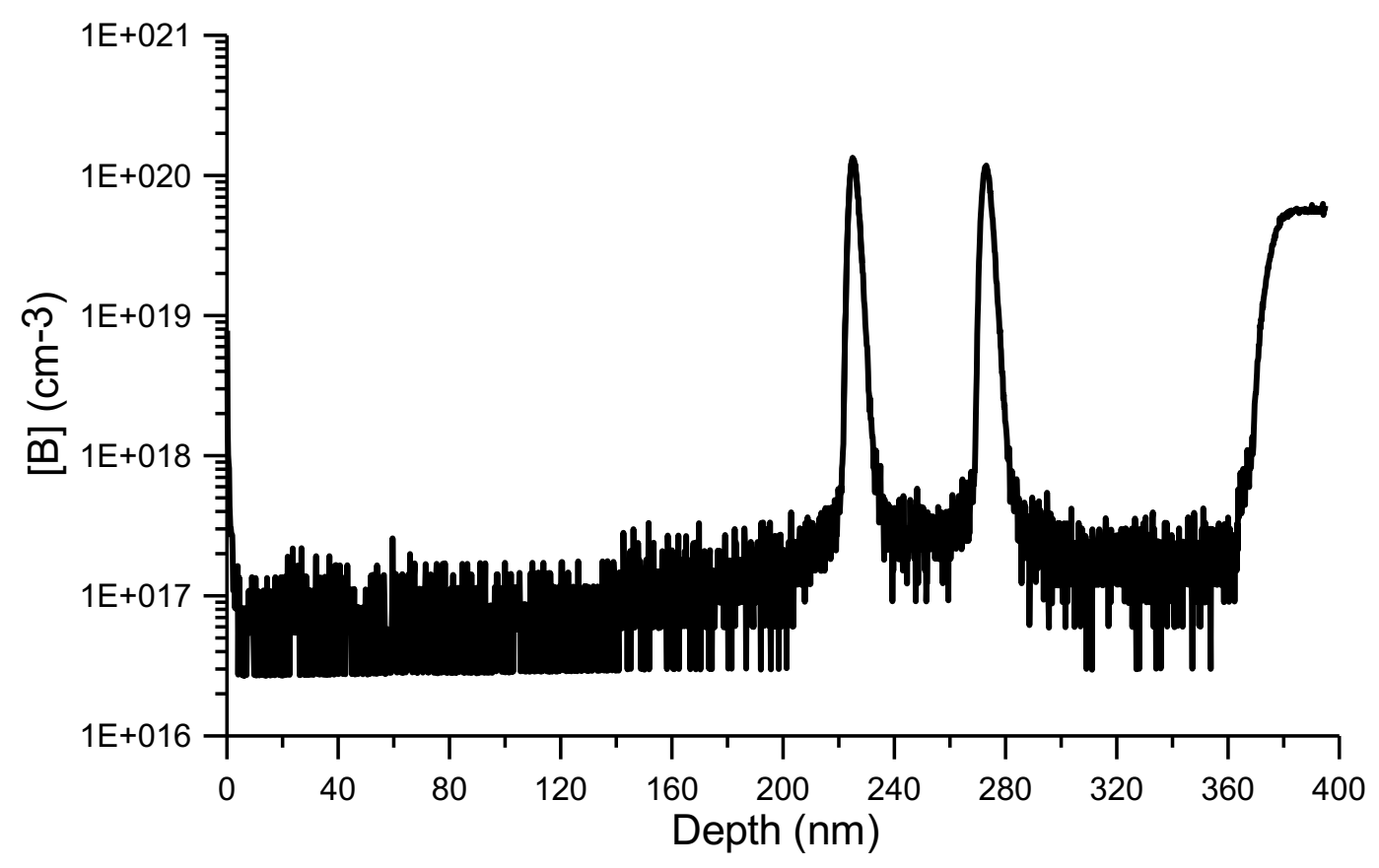

(a)

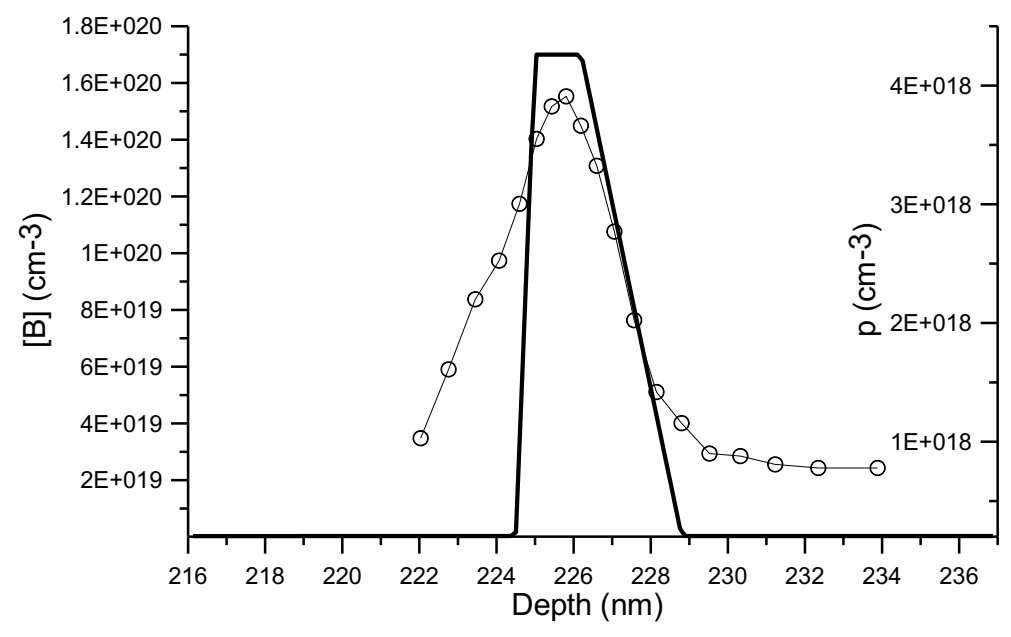

(b)

Figure 3. (a) SIMS-SP of the boron concentration of sample Tr06 containing 2 'delta layers'. The sample was grown on a 3 micron thick heavily boron doped layer grown on a type 1b HPHT substrate; (b) Comparison of boron 
concentration profile recovered using analytical depth resolution function (solid line) and hole profiles extracted from $\mathrm{CV}$ analysis measured at room temperature (circles), Tr06

There are many parallel electrical conduction paths possible in a sample with a capped single delta layer. These consist of conduction on the surface, in the bulk $\mathrm{p}$ - doped capping layer, in the heavily doped 'delta layer', in

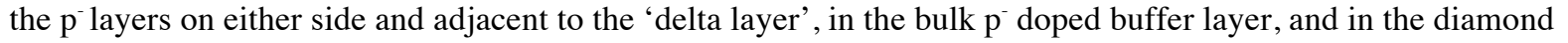
substrate. Surface conduction is easily prevented by oxidation of the diamond surface, but all other paths cannot be ignored. Conduction in the heavily doped 'delta layer' is characterized by low mobilities and high carrier concentrations, typically 3 to $5 \mathrm{~cm}^{2} / \mathrm{Vsec}$ and $10^{20} \mathrm{~cm}^{-3}$. Conduction in the CVD grown bulk p doped layers should have high mobilities, greater than $1000 \mathrm{~cm}^{2} / \mathrm{Vsec}$, but low carrier concentrations and thermal activation energies between 0.2 and $0.37 \mathrm{eV} .{ }^{[2]}$ Conduction in the diamond substrate may vary depending on the unintentional doping of the HPHT substrate with B and N, but generally, these substrates are insulating.

For Hall effect measurements with room temperature sheet carrier concentrations below $10^{12} \mathrm{~cm}^{-2}$, the dominant conduction path is likely the high mobility bulk p buffer and capping layers, while for carrier concentrations above $10^{14} \mathrm{~cm}^{-2}$, the dominant contribution is from conduction in the low mobility, heavily doped 'delta layer'. Hence the region of Table 1 relevant to the enhanced mobility and carrier concentration is the nonitalicized entries between $10^{12}$ and $10^{14} \mathrm{~cm}^{-2}$ sheet carrier concentrations. Sample 1 of ref. had relatively thick delta and buffer $\mathrm{p}^{-}$layers and gave a room temperature mobility of 54.6. Its temperature dependence above $150 \mathrm{~K}$ was dominated by the thermally activated carriers from the bulk $\mathrm{p}^{-}$layers, hence our italicizing its entry in Table $\mathbf{1}$. Note that almost all of our initial Hall effect measurements exceed the previously reported values by over an order of magnitude. It is also clear from the SIMS-SP data (figures 1 and 3) and the CV data (figure 3), that the boron concentrations in the current 'delta doped' layers reported here have not always achieved peak concentrations above the metal insulator transition limit $\left(5 \times 10^{20} \mathrm{~cm}^{-3}\right)$. Hence our measured sheet carrier densities could increase by as much as an order of magnitude in future growths, and thus lower the channel resistance by an order of magnitude.

The Hall effect measurements and the CV data presented here are encouraging indicators of the success of this approach to growth of effective 'delta doped' layers. Yet there is still much work to be done to clearly demonstrate the enhancement necessary for functioning active electronic devices. As pointed out by previous researchers, the Hall effect mobilities overestimate the actual channel mobilities in a device. ${ }^{[14]}$ We are in the process of fabricating and testing Hall Bar structures to more precisely determine the local properties on a scale similar to a device structures. Measuring the temperature dependence (activation energy) of the carrier concentrations will assist in identifying the conduction paths measured by the Hall effect. We are also fabricating and testing various field effect transistor structures to determine actual channel mobilities, conductivity, and gate pinch off. We view the successes presented here as just an initial step toward enabling a class of active electronic devices based on the superlative properties of diamond.

\section{Acknowledgements:}

This work was supported by Act 220 of the Russian Government (Agreement no. 14.B25.31.0021 with the host organization IAP RAS). Specific commercial equipment, instruments, and materials that are identified in this report are listed in order to adequately describe the experimental procedure and are not intended to imply endorsement or recommendation by the National Institute of Standards and Technology (NIST). Alexander Kolyadin and Irakli Aneli of New Diamond Technology (St. Petersburg Russia) are thanked for their assistance in sample preparation technologies. RBJ is grateful to the UKs Engineering and Physical Sciences Research Council (EPSRC) for financial support (EP/H020055/1 and EP/N004159/1) and the EC Horizon 2020 programme for the award of the project 'GREENDIAMOND' (research and innovation programme under grant agreement $\mathrm{N}^{\circ}$ 640947), which also supports the work of ACP-T and M-L H. M-LH acknowledges EPSRC and UCL for the award of a PhD studentship. A.V.D. acknowledges the support of Material Genome Initiative funding allocated to NIST. Arkady Murel is thanked for useful discussion of results and interpretations of CV measurements and P.A. Yunin is thanked for assistance with the SIMS recovery procedure.

\section{References:}


[1] J. E. Field, PROPERTIES OF NATURAL AND SYNTHETIC DIAMOND, Academic Press, London 1992.

[2] M. Gabrysch, S. Majdi, D. J. Twitchen, J. Isberg, Journal of Applied Physics 2011, 109, 4.

[3] P. Muret, P. N. Volpe, T. N. Tran-Thi, J. Pernot, C. Hoarau, F. Omnes, T. Teraji, Diamond and Related Materials 2011, 20, 285.

[4] T. R. Anthony, W. F. Banholzer, J. F. Fleischer, L. H. Wei, P. K. Kuo, R. L. Thomas, R. W. Pryor, Physical Review B 1990, 42, 1104.

[5] R. S. Balmer, J. R. Brandon, S. L. Clewes, H. K. Dhillon, J. M. Dodson, I. Friel, P. N. Inglis, T. D. Madgwick, M. L. Markham, T. P. Mollart, N. Perkins, G. A. Scarsbrook, D. J. Twitchen, A. J. Whitehead, J. J. Wilman, S. M. Woollard, Journal of Physics-Condensed Matter 2009, 21.

[6] M. Werner, R. Job, A. Zaitzev, W. R. Fahrner, W. Seifert, C. Johnston, P. R. Chalker, Physica Status Solidi a-Applied Research 1996, 154, 385.

[7] S. Koizumi, T. Teraji, H. Kanda, Diamond and Related Materials 2000, 9, 935.

[8] T. Klein, P. Achatz, J. Kacmarcik, C. Marcenat, F. Gustafsson, J. Marcus, E. Bustarret, J. Pernot, F. Omnes, B. E. Sernelius, C. Persson, A. F. da Silva, C. Cytermann, Physical Review B 2007, 75.

[9] E. Bustarret, P. Achatz, B. Sacepe, C. Chapelier, C. Marcenat, L. Ortega, T. Klein, Philosophical Transactions of the Royal Society a-Mathematical Physical and Engineering Sciences 2008, 366, 267.

[10] A. Denisenko, E. Kohn, Diamond and Related Materials 2005, 14, 491.

[11] E. F. Schubert, Delta-doping of semiconductors, Cambridge University Press, 1996.

[12] C. I. Pakes, J. A. Garrido, H. Kawarada, Mrs Bulletin 2014, 39, 542.

[13] A. Fiori, J. Pernot, E. Gheeraert, E. Bustarret, Physica Status Solidi a-Applications and Materials Science 2010, 207, 2084.

[14] R. S. Balmer, I. Friel, S. Hepplestone, J. Isberg, M. J. Uren, M. L. Markham, N. L. Palmer, J. Pilkington, P. Huggett, S. Majdi, R. Lang, Journal of Applied Physics 2013, 113.

[15] J. Scharpf, A. Denisenko, C. I. Pakes, S. Rubanov, A. Bergmaier, G. Dollinger, C. Pietzka, E. Kohn, Physica Status Solidi a-Applications and Materials Science 2013, 210, 2028.

[16] G. Chicot, A. Fiori, P. N. Volpe, T. N. T. Thi, J. C. Gerbedoen, J. Bousquet, M. P. Alegre, J. C. Pinero, D. Araujo, F. Jomard, A. Soltani, J. C. De Jaeger, J. Morse, J. Hartwig, N. Tranchant, C. Mer-Calfati, J. C. Arnault, J. Delahaye, T. Grenet, D. Eon, F. Omnes, J. Pernot, E. Bustarret, Journal of Applied Physics 2014, 116, 13.

[17] A. L. Vikharev, A. M. Gorbachev, M. A. Lobaev, A. B. Muchnikov, D. B. Radishev, V. A. Isaev, V. V. Chernov, S. A. Bogdanov, M. N. Drozdov, J. E. Butler, Physica Status Solidi-Rapid Research Letters 2016, 10,324 .

[18] S. C. Eaton, A. B. Anderson, J. C. Angus, Y. E. Evstefeeva, Y. V. Pleskov, Electrochemical and Solid State Letters 2002, 5, G65.

[19] A. B. Muchnikov, A. L. Vikharev, J. E. Butler, V. V. Chernov, V. A. Isaev, S. A. Bogdanov, A. I. Okhapkin, P. A. Yunin, Y. N. Drozdov, Physica Status Solidi a-Applications and Materials Science 2015, 212, 2572.

[20] S. Hofmann, Y. Liu, J. Y. Wang, J. Kovac, Applied Surface Science 2014, 314, 942.

[21] A. Fiori, F. Jomard, T. Teraji, G. Chicot, E. Bustarret, Thin Solid Films 2014, 557, 222; C. Mer-

Calfati, N. Tranchant, P. N. Volpe, F. Jomard, S. Weber, P. Bergonzo, J. C. Arnault, Materials Letters 2014, $115,283$.

[22] G. Chicot, T. Tran Thi, A. Fiori, F. Jomard, E. Gheeraert, E. Bustarret, J. Pernot, Applied Physics Letters 2012, 101, 162101.

[23] R. Edgington, S. Sato, Y. Ishiyama, R. Morris, R. B. Jackman, H. Kawarada, Journal of Applied Physics 2012, 111, 033710. 